\title{
Discrepancias entre los datos ofrecidos por la Secretaría de Salud y la Organización Mundial de la Salud sobre tuberculosis en México, 1981-1998
}

A Renata Báez-Saldaña, MC, M en CM,(1) José Rogelio Pérez-Padilla, MC,(1) Miguel A ngel Salazar-Lezama, MC.(1)

Báez-Saldaña AR, Pérez-Padilla JR, Salazar-Lezama MA. Discrepancias entre los datos ofrecidos por la Secretaría de Salud y la Organización Mundial de la Salud sobre tuberculosis en México, 1981-1998. Salud Publica Mex 2003;45:78-83.

El texto completo en inglés de este artículo está disponible en: http://www.insp.mx/salud/index.html

\section{Resumen}

Objetivo. Describir las tendencias de la morbilidad y mortalidad de la tuberculosis en México, entre 1981 y 1998, comparando datos de la Secretaría de Salud y de la 0 rganizacion Mundial de la Salud. Material y métodos. Se analizó el número de casos y tasas notificados y la tendencia de la enfermedad en los últimos años. Se calculó la incidencia de casos nuevos de tuberculosis baciliferos mediante el riesgo anual de infección tuberculosa, con lo que se estimó el porcentaje de detección de casos bacilíferos en 1997-1998. Resultados. El número de casos de tuberculosis emitido por la 0 rganización Mundial de la Salud supera al notificado por la Secretaría de Salud, discrepancia que se ha reducido. Los casos bacilíferos se han mantenido entre 1993-1998 y se estimó una detección de 66 y de 26\% en 1997 y 1998, respectivamente (para un Riesgo Anual de Infección Tuberculosa de $0.5 \%$ ). La mortalidad se redujo $6.7 \%$ cada año entre 1990 y 1998 mientras que se observó un aumento de casos nuevos, lo que implica la persistencia de la transmisión de la infección entre la población. Conclusiones. Hay discrepancia entre el número de casos de tuberculosis ofrecido por la Secretaría de Salud y la O rganización Mundial de la Salud. De acuerdo con las estimaciones por el Riesgo Anual de Infección Tuberculosa se deja de detectar un número considerable de casos bacilíferos. El texto completo en inglés de este artículo está disponible en: http:// www.insp.mx/salud/index.html

Palabras clave: tuberculosis; epidemiología; notificación de casos; O rganización Mundial de la Salud; mortalidad; México
Báez-Saldaña AR, Pérez-Padilla JR, Salazar-Lezama MA. Epidemiology of Tuberculosis in Mexico, 1981-1998. Inconsistencies between reports from theWorld Health Organization and the Ministry of Health. Salud Publica Mex 2003;45:78-83.

The English version of this paper

is available at: http://www.insp.mx/salud/index.html

\begin{abstract}
A bstract
Objective. To describe the tuberculosis morbidity and mortality trends in Mexico, by comparing the data reported by the Ministry of Health (MH) and the World Health $\mathrm{Or}$ ganization (W HO) between 1981 and 1998. Material and Methods. The number of cases notified in the past few years, their rates, and the trends of the disease in Mexico were analyzed. The incidence of smear-positive pulmonary tuberculosis was estimated for 1997 and 1998 with the annual tuberculosis infection risk (ATIR), to estimate the percentage of bacilliferous cases in 1997-1998. Results. W HO reported more tuberculosis cases for Mexico than the MH. However, this difference has decreased throughout the years. The notification of smear-positive cases remained stable during 1993-1998. The estimated percentages of detection were $66 \%$ for 1997 and 26\% for 1998 (based on ATIR of $0.5 \%$ ).Tuberculosis mortality decreased gradually $(6.7 \%$ per year) between 1990 and 1998, whereas the number of new cases increased, suggesting the persistence of disease transmission in the population. Conclusions. Inconsistencies between case notifications from national data and W HO were considerable, but decreased progressively during the study period. According to ATIR estimations, a considerable number of infectious tuberculosis cases are not detected. The English version of this paper is available at: http:// www.insp.mx/salud/index.html
\end{abstract}

Key words: tuberculosis; epidemiology; notification rates; mortality; world health organization; Mexico

(1) Instituto $\mathrm{N}$ acional de Enfermedades Respiratorias, México, D.F., México.

Fecha de recibido: 9 de octubre de 2001 - Fecha de aprobado: 29 de noviembre de 2002 Solicitud de sobretiros: DraA Renata Báez-Saldaña. Instituto N acional de Enfermedades Respiratorias, Calzada de Tlalpan 4502, Colonia Sección XVI, 14080 México, D.F., México.

Correo electrónico: baezrd@ servidor.unam.mx 
a tuberculosis desde hace mucho tiempo ha constituido un problema de salud pública en el mundo. Actualmente lo sigue siendo, a pesar de los avances para el diagnóstico, prevención y curación de la misma, especialmente en los países en desarrollo. Entre personas de 15 y 49 años de edad causa de 2.4 a 2.9 millones de muertes anuales según varias estimaciones. ${ }^{1}$ Durante el periodo 2000 a 2020 la Organización Mundial de la Salud (OMS) estima que habrán 1000 millones de infectados por Mycobacterium tuberculosis, 200 de los cuales enfermarán y 35 morirán si no se mejora el control de la enfermedad. ${ }^{2,3}$

La tuberculosis resulta de una interacción entre el hombre y el agente causal, Mycobacterium tuberculosis, cuyo crecimiento lento y gran capacidad de adaptación favorecen la persistencia de la infección y la enfermedad en la especie humana.

La exposición se origina en los casos contagiosos (bacilíferos) y la probabilidad de que un contacto se infecte depende de su susceptibilidad y de la magnitud del inóculo. La transmisión es ineficiente ya que se sabe que de los contactos sólo de 25 a 50\% se infectarán,, , 5 y de los infectados sólo 10\% enfermarán en el transcurso de toda la vida. ${ }^{6-8}$ Para que se mantenga la epidemia, se considera que por cada caso contagioso se debe producir un mínimo de uno o dos más de ellos.

Siendo los casos infectantes los que mantienen la transmisión del bacilo de la tuberculosis en la sociedad, la prioridad en los programas de control es su identificación y tratamiento.

Nuestro objetivo fue describir y analizar las tendencias de la morbilidad y mortalidad de tuberculosis en México, de 1981 a 1998, basados en los reportes oficiales de la Secretaría de Salud de México (SSA) y los emitidos por la OMS. Adicionalmente, a partir del riesgo anual de infección tuberculosa (RAIT), se estimaron los casos nuevos bacilíferos y el porcentaje de detección por el programa antituberculoso.

\section{Material y métodos}

Los datos del presente trabajo se obtuvieron de las notificaciones de casos de tuberculosis y de muertes por tuberculosis con sus tasas informadas en México entre 1981 y 1998. Las cifras están reportadas en el Boletín Mensual de Epidemiología de la SSA, en el Registro Nacional de Casos de Tuberculosis, preparado por la Dirección General de Epidemiología (DGE) de la SSA y, a partir de 1995, en el Sistema Unico de Infecciones para la Vigilancia Epidemiológica (DGE). También recolectamos datos de los informes de la OMS, que a su vez, se basan en los datos oficiales que emite el país y que, en principio, deberían coincidir. Para los propósi- tos del presente trabajo, caso de tuberculosis fue aquel notificado oficialmente en México por la SSA, e internacionalmente, por la OMS. Con los reportes descritos se analizaron las posibles discrepancias no sólo en el número de los casos notificados o en sus tasas, sino en la interpretación de la tendencia de la enfermedad en México. Cuando no fue posible obtener cifras en paralelo para todos los indicadores y todos los años, se analizaron solamente los años con información disponible.

Adicionalmente, se estimó la incidencia de casos nuevos de tuberculosis bacilífera con el método RAIT, que se desarrolla a partir de encuestas tuberculínicas e indica la probabilidad de que un sujeto se infecte con Mycobacterium tuberculosis en un año. Estas estimaciones se hacen para tener una idea de la magnitud del subregistro de casos en un país y, en consecuencia, la cobertura y eficiencia del programa antituberculoso. La estimación se realizó para los años 1997 y 1998 considerando un RAIT de 0.5, 1, y 1.5\%, rangos reportados por la OMS/Organización Panamericana de Salud (OPS), para las Américas, 9,10 aunque en 1995 un informe de la OMS lo estimó en $0.6 \%$ para México. Se ha considerado que por cada 1\% de RAIT ocurrirán en promedio 50-60 casos anuales de tuberculosis, con baciloscopía positiva, por cada 100000 habitantes. ${ }^{11} \mathrm{La}$ población total estimada en México en 1997 y 1998 fue de 94281000 y 95830 901, respectivamente, cifras que se utilizaron para las estimaciones correspondientes al RAIT. Se describió la distribución del número de casos notificados de 1993-1998 en función de la edad, y la tendencia de la mortalidad de 1990-1998, con informes de la DGE/ México.

\section{Resultados}

En el cuadro I se muestran los casos notificados de tuberculosis, los bacilíferos y sus tasas por 100000 habitantes de acuerdo con los reportes de la SSA de México y con los de la OMS. Es clara la discrepancia entre los casos notificados y sus tasas cuando se comparan ambas fuentes. La discrepancia es máxima en 1981 y tiende a disminuir con el tiempo, siempre reportando más casos la OMS que la SSA. Esta discrepancia en las tasas y sus tendencias pueden observarse con más claridad en la figura 1, que muestra una tendencia marcadamente descendente en las tasas reportadas por la OMS (salvo un pico en 1997) y valores mucho más estables en los de la SSA.

El cuadro I también muestra los casos bacilíferos y sus tasas por 100000 habitantes de acuerdo con la OMS, con pocas variaciones, salvo por un incremento inusitado en 1997. 
Cuadro I

Casos de tuberculosis en méxico reportados por la Secretaría de salud Y POR LA ORGANIZACIÓN MUNDIAL DE LA SALUD, 1981-1998

\begin{tabular}{|c|c|c|c|c|c|}
\hline $\begin{array}{l}\text { Casos DGE } \\
\text { México }\end{array}$ & $\begin{array}{l}\text { Casos } \\
\text { OMS }\end{array}$ & $\begin{array}{l}\text { Tasa DGE } \\
\text { México }\end{array}$ & $\begin{array}{l}\text { Tasa } \\
\text { OMS }\end{array}$ & $\begin{array}{c}\text { Casos nuevos } \\
\text { baciloscopía positiva (OMS) }\end{array}$ & $\begin{array}{c}\text { Tasa casos nuevos } \\
\text { baciloscopía positiva (OMS) }\end{array}$ \\
\hline 9445 & 32572 & 13.26 & 47.1 & & \\
\hline 8185 & 24853 & 11.19 & 35.1 & & \\
\hline 8724 & 22795 & 11.64 & 31.5 & & \\
\hline 8379 & 14531 & 11.17 & 19.7 & & \\
\hline 7908 & 15017 & 10.17 & 19.9 & & \\
\hline 7008 & 13180 & 9.00 & 17.1 & & \\
\hline 6945 & 14631 & 8.56 & 18.6 & & \\
\hline 11440 & 15371 & 13.83 & 19.2 & & \\
\hline 10078 & 15489 & 11.96 & 19.0 & & \\
\hline 10279 & 14437 & 12.34 & 17.3 & & \\
\hline 9107 & 15216 & 10.71 & 17.9 & & \\
\hline 9274 & 14446 & 10.69 & 16.7 & & \\
\hline 9203 & 15145 & 10.40 & 17.2 & 8164 & 9.3 \\
\hline 10933 & 16353 & 12.13 & 18.3 & 9726 & 10.9 \\
\hline 10273 & 11329 & 11.18 & 12.4 & 9220 & 10.1 \\
\hline 10953 & 10852 & 11.75 & 11.7 & 8495 & 9.2 \\
\hline 12854 & 23575 & 13.57 & 25.0 & 15440 & 16.4 \\
\hline 11371 & 7584 & 11.81 & 7.9 & 6189 & 6.5 \\
\hline
\end{tabular}

D GE = Dirección General de Epidemiología (D GE), México. O MS= la 0 rganización Mundial de la Salud. Los casos se refieren a los notificados con las tasas por 100000 habitantes

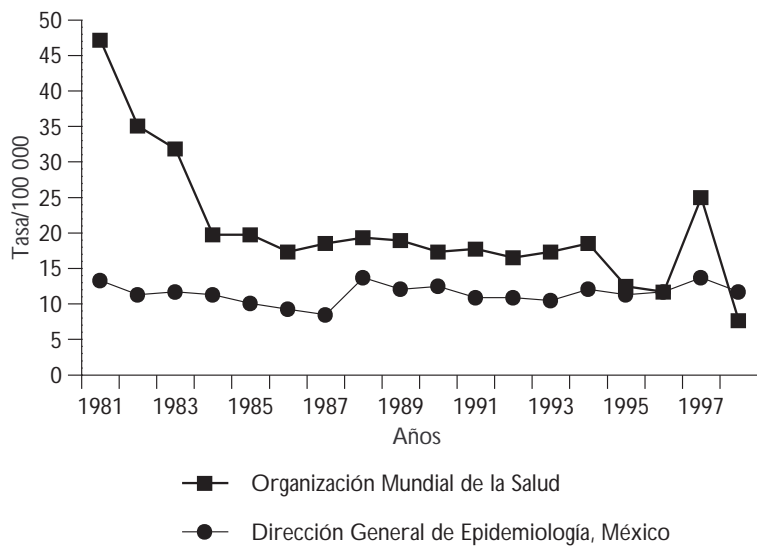

Figura 1.TASa de notificación de tuberculosis en México de acuerdo con la Organización Mundial de la Salud y la Secretaría de Salud.

LA LÍNEA CON CÍRCULOS VACÍOS REPRESENTA LOS DATOS SOBRe MÉxico REPORTAdos POR LA OMS Y LA LÍNEA CON cuadrados negros los reportados por la SecretaRÍA DE SALUd. LAS DISCREPANCIAS SON IMPORTANTES Y EL ORIGEN DE LAS MISMAS NO ES CLARO, YA QUE EN PRINCIPIO AMBOS ORGANISMOS SE BASAN EN LOS MISMOS CASOS
El cuadro II muestra las estimaciones de casos nuevos con baciloscopía positiva de acuerdo al RAIT de $0.5,1$ y $1.5 \%$ para los años 1997 y 1998, junto con los casos notificados, y una estimación del porcentaje de detección. Entre 1997 y 1998 ocurrió un descenso marcado en los casos notificados. Aun considerando un RAIT de $0.5 \%$, se estima una falla de detección considerable de los casos infecciosos. Entre 1993 y 1998 la tuberculosis afectó a todos los grupos etáreos con predominio en jóvenes y adultos; $52.2 \%$ afecta al grupo de 15 a 44 años de edad, $25.4 \%$ a los de 45 a 64 años y el resto, en ambos extremos de la vida (cuadro III). La figura 2 muestra la tasa de mortalidad por tuberculosis obtenidas de la SSA, México, de 1990 a 1998 (por 100000 habitantes), comparada con la tasa de notificación de la misma fuente. En el periodo se aprecia una divergencia entre ambas líneas por un repunte en la tasa de notificación a partir del año 1994.

\section{Discusión}

De acuerdo con los informes de la SSA (cuadro I) el descenso gradual en los casos y en las tasas de notificación que se observó entre 1981-1987, se revirtió a 


\section{Cuadro II \\ EstimaCión de CASOS INFECCIOSOS CON BASE EN EL RIESGO ANUAL DE INFECCIÓN TUBeRCulosA. MéXico, 1997-1998}

\begin{tabular}{|c|c|c|c|c|c|c|c|c|}
\hline \multirow[b]{2}{*}{ Año } & \multirow[b]{2}{*}{ Población* } & \multirow[b]{2}{*}{ Casos notificados* (n) } & \multicolumn{6}{|c|}{ Casos estimados por RAIT* (n) } \\
\hline & & & RAIT $0.5 \%$ & $\%$ detec. & RAIT $1 \%$ & $\%$ detec. & RAIT $1.5 \%$ & $\%$ detec. \\
\hline 1997 & 94281000 & 15440 & 23570 & 66 & 47140 & 33 & 70710 & 22 \\
\hline$\overline{1998}$ & 95830901 & 6189 & 23958 & 26 & 47915 & 13 & 71873 & 9 \\
\hline
\end{tabular}

*RAIT = riesgo anual de infección tuberculosa, \% detec.= porcentaje de casos infecciosos detectados de acuerdo con la tasa de infección calculada por el RAIT con tres niveles

Fuente: 0 rganización Mundial de la Salud

\section{Cuadro III \\ Distribución de La tuberculosis en México POR EDADES ENTRE 1993 Y 1998}

\begin{tabular}{lrc} 
Grupos de edad (años) & $(\mathrm{n})$ & $(\%)$ \\
$<1$ & 37 & 0.36 \\
$1-4$ & 169 & 1.54 \\
\hline $5-14$ & 502 & 4.6 \\
\hline $15-24$ & 1905 & 17.4 \\
\hline $25-44$ & 3805 & 34.8 \\
\hline $45-65$ & 2782 & 25.4 \\
\hline$>65$ & 1336 & 12.2 \\
\hline Ignora & 404 & 3.7 \\
\hline Total & 10940 & 100
\end{tabular}

Promedio del número de casos y porcentaje por grupos de edad de 1993-1998 tal y como lo reporta la Dirección General de Epidemiología, México

partir de 1988 y después se ha mantenido con pocas fluctuaciones. El comportamiento difiere del derivado de los informes de la OMS en los que es notable el declive que ocurrió entre 1981 a 1984 (caída de 55.4\%), con una disminución anual promedio de $22.7 \%$, que excede considerablemente los declives habituales de 10 a $14 \%$ observados cuando los programas de lucha contra la tuberculosis son muy eficaces. ${ }^{12}$ La tasa más elevada de disminución de la incidencia registrada hasta ahora es de 15\% anual en esquimales de Alaska, Canadá y Groenlandia, entre finales de la década de los 50 y la de los $70 .{ }^{13}$ A partir de 1984 las fluctuaciones derivadas del informe de la OMS han sido mínimas, salvo un incremento reportado en 1997.

Estos periodos de estabilidad que alternan con incrementos y declives muy notorios de un año a otro, observables tanto en los informes de la SSA y en los de

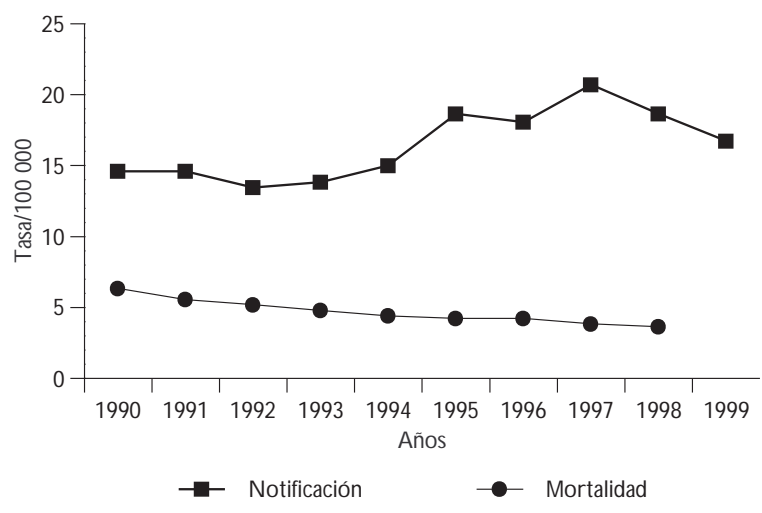

Figura 2.TASA de NOTIFICACIÓN Y DE MORTALIDAD POR TUBERCULOSIS EN MÉXICO.

Se mUestra la tASA POR 100000 habitantes entre 1989 Y 2000 , DONDE ES CLARO EL DESCENSO PROGRESIVO EN LA TASA DE MORTALIDAD, CON UN REPUNTE RECIENTE EN LA TASA DE NOTIFICACIÓN, LO QUE IMPLICA UN INCREMENTO EN LOS CASOS ACTIVOS Y, PRESUMIBLEMENTE, EN LOS CASOS INFECCIOSOS, LO QUE INCREMENTA EL RIESGO DE CONTAGIO EN LA COMUNIDAD

la OMS, más que cambios en la epidemia, muy probablemente expresan diferentes grados de notificación. Por otra parte, las cifras de la OMS y las de la SSA discrepan claramente, lo cual les resta credibilidad. Según el informe de evaluación de la OMS al programa antituberculoso de México en 1995, ${ }^{14}$ existían múltiples instrumentos de registro y notificación de casos, unos recomendados por la Dirección General de Medicina Preventiva (SSA), otros por la Dirección General de Epidemiología (SSA) y otros adoptados por diferentes instituciones o estados, es decir, se carecía hasta 1995 de un sistema único para la notificación y reporte de 
casos. Adicionalmente, no todos los casos tratados se notifican oficialmente. En un estudio realizado en Orizaba, Veracruz, se documentó que $21 \%$ de los casos de tuberculosis diagnosticados y tratados por los diferentes sistemas de salud no eran notificados, ${ }_{1}^{15}$ problema que probablemente se presente en otros lugares del país. Puede suceder también que en los reportes se mezclan los casos prevalentes (fracasos terapéuticos, recaídas y casos crónicos o multifármacorresistentes) con los incidentes, sin una especificación precisa. Es probable que estas y otras inconsistencias e imperfecciones en la notificación tengan que ver con las discrepancias entre los reportes de la OMS y de la SSA, sin embargo, sigue siendo inexplicable por qué hay diferencias si las estadísticas de la OMS sobre México se originan en los reportes ofrecidos por el país. Desconocemos si dentro de la SSA se hayan manejado unas cifras "internacionales", que se ofrecerían a la OMS, y otras "nacionales" o bien, si distintas dependencias dentro de la SSA discrepaban en la estadística. Cualquiera de estas opciones es desorientadora y no debió haberse dado. Afortunadamente parece que se ha eliminado.

Debido a las limitaciones de la notificación se hacen estimaciones basadas en el RAIT, excelente herramienta en los países en desarrollo para evaluar la situación de la tuberculosis, predecir su futura evolución y conocer la eficacia de las medidas de control. Sin embargo, estas estimaciones para ser suficientemente exactas requieren de la realización sistemática de encuestas tuberculínicas, ya que se basan en la prevalencia de la infección tuberculosa. ${ }^{16-18}$ En México no contamos al respecto con datos actuales ni aplicables a todo el país. Los casos bacilíferos entre 1993 y 1998 muestran pocas variaciones salvo un incremento brusco en el número de casos y la tasa respectiva reportada en 1997, que más probablemente se deba a variación en la notificación que en la epidemia. El porcentaje de detección de casos bacilíferos de acuerdo con las estimaciones por RAIT, en general se encuentra por debajo de 70, meta propuesta por la OMS para abatir la epidemia. Esto incluye las estimaciones para un RAIT de $0.5 \%$, cifra optimista que calcula una detección de 66\% en 1977. En 1998 la Organización Panamericana de Salud informó que en la Región de las Américas se detecta $65 \%$ del total de las fuentes de infección. ${ }^{9}$ En la revisión del programa de control de la tuberculosis en México hecha por la OMS en junio de $1995,{ }_{1}^{14}$ se estimó una incidencia anual de 37000 casos en 1993 (IC $95 \%$ entre 30000 y 46000 ) con una tasa de $42 / 100000$ (IC $95 \%$ entre 34 y 51 ) y $13.5 \%$ de casos no tratados (entre $0-28 \%$ ). Los resultados de nuestras estimaciones del número de casos con baciloscopía po- sitiva, realizadas con un RAIT de 0.5 y $1 \%$ son semejantes a los resultados obtenidos en este informe de la OMS, que menciona que las estimaciones para un RAIT de $1.5 \%$ pueden ser representativas de los habitantes de sitios marginados de México.

En México la tuberculosis afecta grupos de todas las edades, pero predomina en jóvenes y en edades medias de la vida, ya que la mitad de los casos tienen entre 15 y 44 años de edad. Esto implica una gran carga social, por afectar a la población económicamente activa, e indica una considerable transmisión del bacilo, con infección reciente que sugiere que la epidemia está activa. ${ }^{13}$

\section{Mortalidad}

En la era previa a la quimioterapia morían de tuberculosis entre 50 a $60 \%$ de los casos. ${ }^{19}$ Sin embargo, desde la introducción de la quimioterapia para el tratamiento de la tuberculosis, se ha disociado la mortalidad por tuberculosis de la aparición de nuevos casos y su curación. Con ello, la mortalidad se ha convertido en un índice poco confiable para evaluar la magnitud del problema y la calidad del tratamiento. ${ }^{1,12,20}$ La certificación de muerte por tuberculosis tiene errores importantes, aun en países desarrollados, con falsos, positivos y negativos. En países industrializados la mortalidad por tuberculosis disminuye cada año entre $4-5 \%$ aun sin tratamiento, este es el ritmo de eliminación espontánea que puede incrementarse hasta en $7-9 \%$ con quimioterapia efectiva y hasta $14 \%$, con muy buenos programas de control. En México observamos una tasa de disminución anual promedio de $6.7 \%$. En la figura 2 se observa la disminución paulatina y sostenida en la mortalidad, sin embargo la tasa de casos se aprecia con un repunte, lo que sugiere que muchos enfermos no se curan y pueden permanecer infecciosos perpetuando la transmisión de la infección entre la población.

La estrategia de tratamiento acortado, estrictamente supervisado (TAES) para la tuberculosis es una de las intervenciones de salud más costo efectivas. En 1995 permitió una tasa de curación de $78.5 \%$, comparado con $44.8 \%$ en pacientes tratados en áreas sin TAES. ${ }^{21}$ En algunos paises se han observado mayores tasas de curación. ${ }^{22}$ Desafortunadamente, en México, en el año 1997, sólo 8\% de la población estaba cubierta bajo esta estrategia, aunque para el año 1998 se había incrementado a $67 \%$ de acuerdo con los datos de la OMS.

La infección por el VIH es el mayor riesgo conocido para desarrollar tuberculosis teniendo infección previa. El riesgo es de 5-15\% anual, comparado con $10 \%$ en toda la vida que presenta un sujeto sin infec- 
ción por el VIH. ${ }^{23}$ En México, la coinfección tuberculosis-VIH es de aproximadamente $3 \%$ y se estima que $25 \%$ de los sujetos infectados por el VIH desarrollan tuberculosis clínica durante el curso de su infección. ${ }^{24}$

La fármacorresistencia del bacilo tuberculoso va en aumento sobre todo en algunas regiones del mundo, por la utilización inapropiada de los esquemas de tratamiento, que seleccionan cepas resistentes de $M y$ cobacterium tuberculosis. La tasa de curación de una tuberculosis fármacosensible es mayor a $95 \%$ y disminuye a $50 \%$ o menos en los casos de multifármacorresistencia. A escala mundial hay variaciones muy amplias de las tasas de prevalencia de fármacorresistencia. De acuerdo con cifras basadas en los informes de un estudio global realizado de 1985 a 1994 por la OMS/UICTER (Unión Internacional contra la Tuberculosis y Enfermedades Respiratorias), la resistencia primaria a isoniacida oscila entre $0-16.9 \%$, y para rifampicina entre 0-3\%. En México se efectuó un estudio de fármacorresistencia en 1997 en los estados de Baja California, Sinaloa y Oaxaca. La resistencia a uno o más fármacos de primera línea en casos nuevos fue de $12.9 \%$, y en los casos de retratamiento de $50.5 \%$, y la multifármacorresistencia se observó en el $2.4 \%$ de los casos nuevos y en $22.4 \%$ de los de retratamiento. ${ }^{25}$

\section{Conclusiones}

Observamos que la OMS ha reportado más casos de tuberculosis en México que los notificados por la Secretaría de Salud. El origen conviene que se aclare aun cuando la diferencia ha disminuido progresivamente. En general, la mortalidad por tuberculosis ha disminuido paulatinamente mientras que la aparición de casos nuevos ha tenido un repunte, lo que implica un incremento en la población de tuberculosos activos potencialmente infectantes. Carecemos de encuestas nacionales tuberculínicas que permitan mejores estimaciones del riesgo anual de infección tuberculosa, de los casos nuevos esperados y del porcentaje de detección de los casos bacilíferos. Las estimaciones con los datos existentes sugieren que un número importante de tuberculosos bacilíferos no se detecta.

\section{Referencias}

1. Enarson DA. Commentary. Why not the elimination of tuberculosis. Mayo Clin Proc 1994; 69:85-86.

2.W orld Health 0 rganization. G lobal tuberculosis control, communicable diseases. Ginebra: 1999.
3.W orld Health 0 rganization. W hat is DOTS?:A guide to understanding the W HO -recommended TB control strategy known as DOTS. Ginebra: W HO, 1999.

4. Grzybowski S, Barnett GD, Styblo K. Contacts of cases of active pulmonary tuberculosis. Bull Int Union Tuberc Lung D is 1975; 50:90-106.

5. Shaw JB,W ynn-W illiams N . Infectivity of pulmonary tuberculosis in relation to sputum status. Am Rev Tuberc 1954; 69:724-732.

6. Rieder HL. Bases epidemiológicas del control de la tuberculosis. París: Unión Internacional contra laTuberculosis y Enfermedades Respiratorias. 1999.

7. Friedman LN .Tuberculosis. Current concepts and treatment. N ew Haven (CT): 1994:286-301.

8. American Thoracic Society. Diagnostic standards and classification of tuberculosis in adults and children. Am J Respir Crit Care Med 2000; 161:1376-1395.

9. 0 rganización Panamericana de la Salud. Informe. El control de laTuberculosis en las A méricas. W ashington, D.C.: O PS, 2000.

10. Raviglione MC, Snider DE Jr, Kochi A. G lobal epidemiology of tuberculosis, morbidity and mortality of a worldwide epidemic. JAMA 1995; 273:220-226.

11. Styblo K. Relación entre riesgo de infección tuberculosa y riesgo de desarrollar una tuberculosis contagiosa. Bol Union Int Tuberc Enf Resp 1985; 60:117-119.

12. C aminero LJA. Medidas básicas para el control de la tuberculosis en una comunidad. Med Clin 1994; 102:67-73.

13. G rzybowski S.A propósito de la historia natural de la tuberculosis. Bol Union Int Tuberc Enf Resp 1991;66:213-214.

14.W orld Health 0 rganization. Tuberculosis Control in Mexico:Joint Programme Review. Ginebra:W HO, junio 1995.

15. García-García ML, Small PM, García-Sancho C, Mayar-Maya ME, Ferreyra-Reyes L, Palacios-Martínez $M$ et al.Tuberculosis epidemiology and control in Veracruz, Mexico. Int J Epidemiol 1999; 28:135-140.

16. C aminero LJA. Editorial. Situación actual de la tuberculosis en España. Arch Bronconeumol 1994; 30:371-374.

17. Bleiker MA, Sutherland I, Styblo K, D am HG, Misljenovic O. N ormas para la estimación del riesgo de infección tuberculosa según los resultados de la prueba tuberculínica en una muestra representativa de niños. Bol Union Int Tuberc Enf Resp 1989; 64:7-12.

18. Sutherland I.Acerca del riesgo de infección. Bol Union Int Tuberc Enf Resp 1991; 66:209-211.

19. Murray CJL, Styblo K, Rouillon A. Tuberculosis in developing countries: Burden, intervention and cost. Bull Int U nion Tuberc Lung $D$ is 1990; 65: 6-24.

20. Grzybowski S, Enarson DA. The fate of cases of pulmonary tuberculosis under various treatment programmes. Bull Int Union Tuberc Lung Dis $1978 ; 53: 70-75$.

21. Smith I.The Stop TB Initiative.A partnership for action against tuberculosis. Tub Ind J 1999; 46(81):1-13.

22. Cao JP, Zhang LY, Zhu JQ, Chin DP. Two year follow-up of directlyobserved intermittent regimens for smear positive pulmonary tuberculosis in China. Int J Tuberc Lung D is 1998; 2:360.

23. $\mathrm{N}$ arain JP, Raviglione MC, Kochi A. HIV-asso ciated tuberculosis in developing countries: Epidemiolgy and strategies for prevention. Tuberc Lung $D$ is 1992; 73:311-321.

24.Angeles-Angeles A, Gassman N . Comparative demographic and autopsy findings in acquired immunodeficiency syndrome in two Mexican populations. J A cquir Immune D efic Syndr 1990; 3:579-583.

25. G ranich RM, Balandrano S, Santella AJ, Binkin N J, C astro KG, MárquezFiol A et al. Survey of drug resistance of Mycobacterium tuberculosis in 3 Mexican states, 1997. Arch Intern Med 2000; 160:639-644. 